\title{
A Neutrophil's Perspective: the Innate Response to Tuberculosis Infection and the Induction of Adaptive Immunity
}

\author{
Lorinda du Toit, Willem J du Plessis and Andre G Loxton* \\ SA MRC Centre for TB Research, Stellenbosch University, South Africa
}

Submission: January 26, 2017; Published: April 24, 2017

*Corresponding author: Andre G Loxton, SA MRC Centre for TB Research, Stellenbosch University, South Africa, Tel: (+27) 21 938 9399; Fax: (+27) 2186567 8452; Email: GL2@sun.ac.za

Abstract

Neutrophils were traditionally viewed as short lived, terminal, innate effector cells that eliminate microbes and remove cellular debris at the site of infection or inflammation. They mediate this by phagocytosis, the release of reactive oxygen species, antimicrobial proteins and proteolytic enzymes. During recent years, studies have demonstrated that they are longer lived than initially thought and they mediate a large number of immune functions by the release of a variety of preformed and newly synthesized molecules such as cytokines and chemokines. In this review we will reconsider the mechanism by which neutrophils operate, especially focusing on the response to tuberculosis infection and we will look at a recent study indicating neutrophils as sophisticated mediators of innate and adaptive immune responses.

Keywords: Neutrophils; Activation; Neutrophil extracellular trap; Apoptosis

\section{Introduction}

Immunity is defined as a host's defence mechanism against disease. The significance of the immune system for health is gravely illustrated by the frequent observation of disease and infection in individuals with inadequate or faulty immune responses. The host's mechanism of defence consists of innate immunity, present in all healthy individuals as the first line of defence against infections; and adaptive immunity, which develops more gradually and provides specific and more specialized defence against pathogens. The importance of the immune system for host protection against infection was intensely highlighted during the advent of AIDS (acquired immunodeficiency syndrome) during the 1980 's. AIDS is a spectrum disease caused by an infection with HIV (human immunodeficiency virus) [1]. As the disease progresses, the immune system steadily declines, resulting in an increased susceptibility to opportunistic infections that can become life threatening; the reactivation of latent infections such as tuberculosis; and greater incidence of several cancers. In individuals with a healthy immune system, latent tuberculosis are not eradicated but are constrained by intact immune responses. Tuberculosis is caused by infection of Mycobacterium tuberculosis (M. tb), an intracellular bacterium [2]. According to the World Health Organization during 2013 tuberculosis accounted for about 1.3 million deaths on average
[3]. Today, one of the most prominent threats in the abolishment of the tuberculosis epidemic is multidrug resistant M. tb (MDRTB), which developed due to extensive and uncontrolled use of antibiotics. It is evident from these statistics that more effective tuberculosis treatments and diagnostic tools are required.

Neutrophils, being the specialized front-line fighters, arrive at the scene within minutes after a breach of immunity. They are directly associated with inflammation and react vigorously against pathogenic infection, often leaving behind a trail of immunopathology. Neutrophils instruct monocytes, dendritic cells and other lymphocytes and aid as a direct connection between innate and humoral immune responses. In this review we reassess the duty of the innate immune system, especially focusing on the role neutrophils play during tuberculosis infection. We will also consider a pioneering study led by Andrea Cerutti et al. [4] in uncovering novel communications between different divisions of the immune system.

\section{The phenotype and origin of neutrophils}

Neutrophils are effector cells that form part of the innate immune system. They are also known as polymorphonuclear neutrophils (PMN's) due to their lobe shaped nuclei. Together with eosinophils and basophils they form part of the granulocyte 
cell family. The cytoplasmic granules contained within neutrophils are characterized by their ability to not take on basophilic (blue) or acidophilic (red) dye stains, they instead colour pale pink during blood smear stains [4]. These native myeloid cells are formed in the bone marrow where growth factors and cytokines instruct pluripotent hematopoietic cells to differentiate into myeloblasts. These myeloblasts are mouldable cell types dedicated to develop into granulocytes. During neutrophil development, protein-containing cytoplasmic granules are formed and released into circulation following their maturation [4]. The strictly monitored process by which matured neutrophils are released from the bone marrow are regulated by cytokines and chemokines. Stimulation to release neutrophils into circulation (from the bone marrow) is governed by the SDF-1 $\alpha /$ CXCR4 chemokine axis, which also maintains an assemblage of neutrophils to promote rapid release should an infection arise [5]. Between 50-70\% of the white blood cell population is represented by neutrophils, making them the most abundant white blood cell type. They are highly mobile and found dispersed in tissues, but are predominantly found in areas of acute inflammation and severe necrosis.

\section{The neutrophil mode of action}

Neutrophils are the first line of defence against infection and migrate to the site of inflammation or tissue damage within minutes following trauma. These innate immune cells are the first to be activated and a key attribute of acute inflammation [6]. Neutrophils undergo a process of degranulation following activation and release into circulation. During this process an extensive amount of membrane delineated granules release their payload consisting of potent anti-microbial agents, such as alkaline phosphatase- containing granules, specific granules and azurophil granules. Azurophil granules include ionic granule proteins such as "lysosomal enzymes" and defensins. Defensins are antimicrobial peptides capable of inserting themselves into microbial cell membranes via electrostatic interactions [7] or through transmembrane potential driven insertions [8]. The membrane insertion brings about a change in the permeability of the membrane, and ultimately results in demise of the microbe. Defensins are effective against a wide array of organisms including bacteria, fungi and even viruses [9]. These anti-microbial peptides further add to the immune response by scrupulously inducing the migration of CD4+/CD45RA and CD8+ T-cells in humans and also serve as a chemo tactic for immature dendritic cells derived from either peripheral blood monocytes or CD34+ progenitors [10].

Neutrophils also have the ability to produce toxic oxygen species that include hydrogen peroxide, hydroxyl radicals and superoxide anions. These reactive oxygen species (ROS) have numerous functions, including acting as cellular messengers [11], regulating the apoptotic process of neutrophils [12] and modulating other reactive immune cells [13]. The microbicidal role of oxygen derived free radicals are emphasized by their ability to promote lipid peroxidation, DNA damage and the oxidation of proteins, resulting in cell death [14].

Even though neutrophils are the chief motive of the innate immune system, in humans they only spend an average of 5 and half days in circulation [15]. The brief lifespan of neutrophils compared to other innate immune cells, could be attributed to their arsenal of anti-microbial compounds that could induce severe immunopathology and cause serious harm to the host if released unrestrained. Constitutive apoptosis is another mechanism used to control neutrophil numbers, to regulate the inflammatory potential of these innate cells [16]. This essential process maintains the delicate balance between neutrophils behaving as effectors during host defence and neutrophils functioning as inducers of immunopathology. Abadie and colleagues [17] infected mice with a genetically modified strain of Mycobacterium bovis bacilli Calmette-Guerin (BCG) that express an enhanced green fluorescent protein (EGFP). Co-expression of EGFP enabled them to determine the essential role neutrophils play in the capture and transport of rBCG-egfp to the secondary lymphoid organs, including Peyer's patches, lymph nodes and spleen. Their results showed that neutrophils can also play a part in antigen presentation in vivo. The conclusion was that neutrophils have the capacity to exit the site of infection via the afferent lymphatic system, migrate to the secondary lymphoid tissue and take part in the transport and presentation of live microbes [17].

The release of cytokines and chemokines recruit neutrophils to the site of infection. After an encounter and subsequent infection by $M$. $t b$, macrophages produce interleukin-8 (CXCL8 or IL-8) [18]. With regards to neutrophil immunity, CXCL8 is one of the most influential chemokines [19]. Neutrophils possess a high number of chemokine receptors that are specific for CXCL8. It serves as a chemo attractant and potent angiogenic factor, crucial for activation and recruitment of neutrophils [19]. A significant correlation exists between the amount of CXCL8 protein present and the number of neutrophils accounted for [20]. Activated human CD4+ T-cells secrete interleukin-17 (IL17). The cytokine, IL-17 is responsible for inducing an elevated concentration and an increased release of CXCL8 from human bronchial epithelial and venous endothelial cells. It was further illustrated that in vivo, after intra-tracheal addition of hIL-17 (human interleukin-17), neutrophils were selectively recruited to the airways of the rats. Laan et al. [21] established that there exists a link between neutrophil recruitment and T-lymphocytes by demonstrating that hIL-17 mobilizes neutrophils to the site of infection via the release of CXC chemokines [21].

\section{The immune response to intracellular pathogens- a neutrophil's perspective}

During infection neutrophils move to the area of infection where they attempt to kill the intruding micro-organism by phagocytosis followed by exposure to ROS and other antimicrobial metabolites. The measure of resistance that the host 
will have against various bacterial and fungal infections is also determined by neutrophils. Elevated chemotaxis accompanied by an increased accumulation of neutrophils in the granuloma suggests another role of these innate cells during $M$. $t b$ infection. By activating DC's (dendritic cells), neutrophils also act as a messenger between the innate and specific acquired immune system [22]. Neutrophils have also shown to enhance immunity. When apoptotic neutrophils, infected with mycobacteria, are phagocytosed by macrophages, the acquired neutrophil granules add to the increased microbicidal effect that these macrophages have against the bacteria $[19,21]$. After inhalation of $M$. $t b$, neutrophils and macrophages are of the first cells that come into contact with the bacteria. Macrophages, being a substantial source of CXCL8, are responsible for the increased recruitment of neutrophils to the site of infection. As a result, the newly recruited neutrophils produce cytokines like TNF- $\alpha$ (tumour necrosis factor-alpha) which have a paracrine effect on macrophages [19]. This illustrates that the immune system is interlinked in function, and how various components influence one another to shape the type of immune response that is elicited [23].

Because of their notorious association with immunopathology recognized during acute infection, most data pertaining to neutrophils aim attention at the intracellular killing mechanism and overlook their probable extracellular activities. In recent years [24], described an extracellular, neutrophil-mediated antimicrobial mechanism to contain and kill micro-organisms. This mechanism involves the formation of neutrophil extracellular traps (NETs), as shown in Figure 1, which consist of chromatin, lined with anti-microbial proteins. These proteins are granular in nature and have the ability to contain and kilo gram-positive bacteria [25,26], gram negative bacteria [27] and even fungi [28]. NADPH oxidase, coupled with NET formation, produce ROS responsible for induction of a cell death process unique from necrosis or apoptosis.

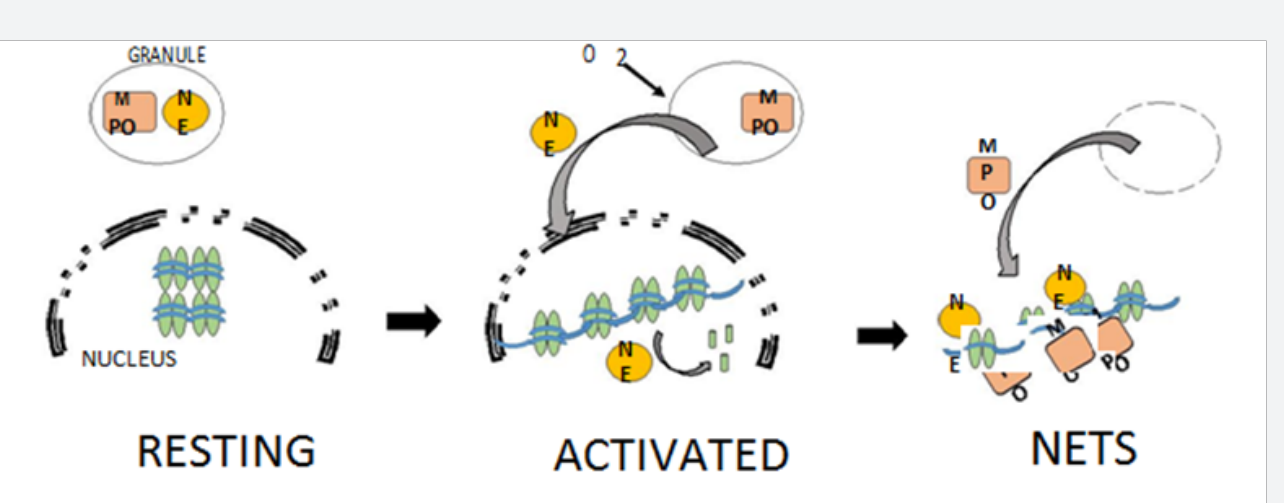

Figure 1: An example of the formation of the neutrophil extracellular trap (NET). The resting neutrophils contain azurophilic granules with neutrophil elastase (NE) and Myeloperoxidase (MPO). Activated neutrophils produce ROS, NE leaks from the granule and moves to the nucleus where it slices histones and aids chromatin decondensation. NE and MPO boost chromatin decondensation and results in the rupture of the cell to release the NET. Adapted from [78].

\section{Phagocytosis, cellular cross-talk and the induction of the adaptive immunity}

Neutrophils remain dormant in circulation until they encounter an infectious agent, this is followed by a phase of activation that promotes and enhances inflammatory responses and anti-microbial action [29]. This phase of activation includes various stages of phenotypical and functional changes in neutrophils. The cell surface receptors employed by neutrophils in the interaction with microbes are altered by low levels of activating agents. Direct recognition, as well as opsonisation, the process by which opsonins coat infecting bacteria making them more prone to be phagocytosed, seems to play a key role in the process of mycobacterial internalization. Alemán and colleagues [30] showed that mice deficient in Toll like receptor 2 (TLR2) had reduced control over both M. avium and $M$. tb infections, highlighting a role for TLR2. Toll-like receptor 4 (TLR4) should not be neglected, as Godaly [31] illustrated that when TRL4 was blocked, CXCL8 production in response to BCG infection was significantly reduced. Bacteria are phagocytosed once recognition has taken place. Neutrophils play a central role in phagocytosis, the primary mechanism by which pathogens and cell debris are removed from the body. Phagocytosis is a dynamic process mediated by cell receptors. The internalization of microbes occurs through the cell membrane into vacuoles called phagosomes. Inside these vacuoles, microbes are exposed to various anti-microbial peptides and degradative molecules. The type of interaction between the microbe and the neutrophil determine the specific mechanism of internalization that is utilized. As soon as neutrophils exit the circulatory system and enter the site of infection, they interact with invading pathogens and become activated. They are able to partake in specific antimicrobial actions upon stimulation by certain chemokines and cytokines.

With their rapid action against infection, it is evident that neutrophils play an essential part in creating the optimal environment for the host to reciprocate with a suitable adaptive 
immune response. This is accomplished by using chemokines and cytokines as mediators to issue instructions to virtually all other types of immune cells. This is a critical process for the development of an appropriate inflammatory response. Neutrophils maintain a low transcriptional signature during their inactive state in the circulatory blood and only once they encounter infection do they experience an immense transcriptional burst and successive activation which results in the formation of signaling compounds [32,33].

Even though, compared to other immune cells, neutrophils do not produce a large amount of cytokines per cell, at the site of infection or inflammation they are plentiful and present in large numbers, making their relative contribution rather significant [34]. Since the primary response of these innate cells is to boost their numbers, CXCL8 is most abundantly produced due to its main function being to recruit more neutrophils [35]. In addition to chemokines and cytokines, a variety of other signaling compounds are secreted by neutrophils. These include granule content [36], lipids [37], hydrogen peroxide (ROS) and some mediators by means of cell to cell contact [38]. Other leukocytes, such as macrophages, cooperate with neutrophils to combat various pathogenic infections. Using immunohistochemistry, Ramos-Kichik et al. [24] noticed that following a mycobacterial infection, macrophages contained granulocytes. This adequately illustrated that through phagocytosis, macrophages obtained lactoferin, a protein produced by granulocytes such as neutrophils and inherently not present in macrophages. Previously, the presence of neutrophils during mycobacterial infections was thought of as inconsequential and temporary, however, these findings significantly highlighted the function of these leukocytes [23]. Dendritic cells (DCs), classified as probably the most important antigen-presenting cells, have the capacity to capture and present antigens in the secondary lymphoid tissue. They also release interleukin-12 (IL-12) which has been demonstrated to be integral in the stimulation of a $\mathrm{T}$ helper 1 (Th1) directed cytokine response [39]. Van Gisbergen et al. [38] confirmed that active neutrophils, both in vitro and in vivo, robustly cluster with and activate the maturation of DCs. This facilitates them to set off a strong T-cell response directed at a type $1 \mathrm{~T}$-cell polarization. This DC-neutrophil interaction is aided by the binding of C-type lectin unique to DCs (DC-SIGN), to Mac-1 [38]. The interaction of immature DCs with neutrophils may, in distant lymph nodes, modulates immune responses. Bennouna et al. [40] confirmed this with data from a murine study, showing that both DC maturation and cytokine production was induced by neutrophil derived TNF- $\alpha$. Neutrophils have also been shown to, in vitro, collaborate with natural killer (NK) cells and DCs. The study by Costantini et al. [41] f confirmed that neutrophils, using CD18-ICAM-1 interactions, very specifically communicate with DCs. This correspondence promotes the production of IL-12p70 by DCs, which in turn, results in the stimulation of Interferon- $\gamma$ (INF- $\gamma$ ) production by NK cells and eventually furthers the activation of neutrophils, culminating in a positive feedback loop. Simultaneously, by direct binding (in a cell to cell manner), NK cells become further activated by neutrophils [41].

\section{Neutrophil-associated Chemokines and cytokines}

By means of cytokine and pattern-recognition receptors, neutrophils may be directly activated to secrete immunomodulatory elements [42]. Surprisingly, even cathelicidins and defensins (anti-microbial peptides found inside the granules of neutrophils) have the capacity to be immune-modulatory. Table 1 outlines the function of some of this immune response modulating cytokines.

Table 1: Cytokines and chemokines released by neutrophils during a host immune response, their regular function and the role they play during M.tb infection.

\begin{tabular}{|c|c|c|}
\hline $\begin{array}{c}\text { Cytokine/chemokine } \\
\text { name }\end{array}$ & Function & Role during Tuberculosis infection \\
\hline Interleukin-1 (IL-1) & $\begin{array}{l}\text { Actively induces fever. It is also responsible } \\
\text { for the development of inflammation [43], } \\
\qquad[44] \text {. }\end{array}$ & $\begin{array}{l}\text { Because the signaling of IL- } 1 \text { is a crucial factor of the MyD88- } \\
\text { dependent innate immune response to } M \text {. th, it is paramount in the } \\
\text { successful resistance, in vivo, against } M \text {. tb infection. The MyD88- } \\
\text { dependent response occurs via a caspase-1 independent mechanism } \\
\text { [45]. }\end{array}$ \\
\hline Interleukin-6 (IL-6) & $\begin{array}{l}\text { Is versatile with a plethora of activities. Its } \\
\text { functions include the terminal maturation } \\
\text { of B-cells, the promotion of B-cell antibody } \\
\text { production and acting as a growth } \\
\text { stimulating molecule on hematopoietic } \\
\text { progenitors in conjunction with IL-1 and } \\
\text { IL-3 [46]. }\end{array}$ & $\begin{array}{l}\text { IL- } 6 \text { is vital for the activation of macrophages and the generation of } \\
\text { pro-inflammatory responses. } M \text {. } t b \text { inhibits the production of IL- } 6 \\
\text { by means of a stress response factor Sigh. This suggests that IL-6 } \\
\text { plays an essential part in the acquired immunity against } M \text {. tb. IL- } 6 \text { is } \\
\text { fundamental in the generation of a protective Th1 immune response } \\
\text { against } M \text {. } t b \text { after vaccination with a subunit vaccine. }\end{array}$ \\
\hline
\end{tabular}




\begin{tabular}{|c|c|c|}
\hline $\begin{array}{l}\text { Interleukin-8 (IL-8 / } \\
\text { CXCL8) }\end{array}$ & $\begin{array}{l}\text { Induces chemotaxis (by means of a } \\
\text { feedback loop, more neutrophils get } \\
\text { recruited to the site of infection), } \\
\text { respiratory burst and exocytosis. } \\
\text { Additionally, CXCL8 up regulates } \\
\text { complement receptors } 1 \text { and } 3 \text { [47]. } \\
\text { Interleukin-1 receptor antagonist } \\
\text { [48]-proving to play a regulatory part } \\
\text { during inflammation by inhibiting (both in } \\
\text { vitro and in vivo) effects of IL-1 [49]. }\end{array}$ & $\begin{array}{l}\text { Lung epithelial cells release IL- } 8 \text { after stimulation by } M . t b \text {. } \\
\text { Neutrophil recruitment to the site of infection is increased when } \\
\text { IL-8 levels are elevated. Even though this enhanced trafficking } \\
\text { of neutrophils might be involved in the clearance of } M \text {. th, tissue } \\
\text { damage may result due to released proteases and oxidants when } \\
\text { neutrophils are present in excess. }\end{array}$ \\
\hline $\begin{array}{l}\text { Tumour necrosis factor- } \\
\alpha(\text { TNF- } \alpha)\end{array}$ & $\begin{array}{l}\text { Shows a wide spectrum of effects. } \\
\text { It inaugurates fever [50], changes } \\
\text { the production of collagenase and } \\
\text { prostaglandin E2 by fibroblasts } \\
\text { [51], stimulates the synthesis of } \\
\text { prostaglandin E2 and IL-1 in resting } \\
\text { macrophages [52],initiates osteoclastic } \\
\text { bone resorption [53], has the capacity } \\
\text { to inhibit lipoprotein lipase [54] and to } \\
\text { function cooperatively with other cytokines } \\
\text { including IL-1 [55]. }\end{array}$ & $\begin{array}{l}\text { The methodical induction of macrophage apoptosis after bacillary } \\
\text { infection is a mechanism by which TNF- } \alpha \text { is believed to mediate } \\
\text { a successful host immune response to mycobacteria. Macrophage } \\
\text { apoptosis is an important component of granulomas associated with } \\
\text { tuberculosis and might assist in } \\
\text { maintaining their integrity }\end{array}$ \\
\hline $\begin{array}{l}\text { Macrophage inflammatory } \\
\text { protein (MIP) }-1 \alpha / \beta\end{array}$ & $\begin{array}{l}\text { An inducible protein partaking in various } \\
\text { pro-inflammatory activities. Subsets of } \\
\text { leukocytes, such as neutrophils, can be } \\
\text { recruited to the site of inflammation by } \\
\text { monocyte chemo attractant protein (MCP)- } \\
1[49] .\end{array}$ & $\begin{array}{l}\text { Because of the chemo attractant property of macrophage } \\
\text { inflammatory proteins, they are likely to play a considerable part } \\
\text { in respiratory tract defences during infections such as tuberculosis } \\
\text { [56]. }\end{array}$ \\
\hline $\begin{array}{l}\text { Growth regulated alpha } \\
\text { protein }(\text { GRO- } \alpha)\end{array}$ & $\begin{array}{l}\text { An inducible protein partaking in various } \\
\text { pro-inflammatory activities. Subsets of } \\
\text { leukocytes, such as neutrophils, can be } \\
\text { recruited to the site of inflammation by } \\
\text { monocyte chemo attractant protein (MCP)- } \\
1 \text { [49]. Is produced in guinea pigs, mice } \\
\text { and humans [57], [58], [59] and believed } \\
\text { to be a member of the pro-inflammatory } \\
\text { group of cytokines. It also contributes to the } \\
\text { inhibition of apoptosis. }\end{array}$ & $\begin{array}{l}\text { The chemokines secreted by neutrophils may play an essential } \\
\text { part in the development and advancement of } M \text {. tb inflammation. } \\
\text { It has been validated, in vitro and in vivo, that neutrophils } \\
\text { produce cytokines in response to M. avium, M. bovis BCG and M. tb } \\
\text { stimulation [58], } \\
\text { [60]. }\end{array}$ \\
\hline
\end{tabular}

\section{Neutrophils activating B-cells : a special focos on the study by Puga et al. [4]}

Collaboration between the innate and adaptive arms of the immune system is required for the successful eradication of pathogens. While the innate branch launches a rapid, less specified response against pathogens by recognizing conserved microbial patterns, the adaptive response is highly specific and somewhat delayed, taking days to become apparent. The participation of innate immune cells in the mediation of B-cell responses has been generally restricted to opsonisation and phagocytosis of pathogens coated with antigens. However, studies by Chen et al. [61] and Chu et al. [62] illustrated that innate immune cells, basophils and eosinophils respectively, secrete factors such as interleukin-6 (IL-6), a proliferationinducing ligand (APRIL), B-cell-activating factor of the TNF family (BAFF), which stimulate the activation of B-cells. Similarly, it has been shown that neutrophils impact adaptive immune responses during infection by regulating the activation of dendritic cells by way of interleukin-10 [63] and alarmins [36]. However, it has been largely unknown how neutrophils regulate a response in the humoral branch of the adaptive immune system. In a pioneering study by Andrea Cerutti, the authors demonstrated that splenic neutrophils have the capacity to act as proficient helper cells for splenic marginal zone (MZ) B-cells, resulting in the generation of matured antibodies with increased affinity for a specific antigen (fully illustrated in Figure 2. The study commenced by analyzing neutrophil distribution in tissue sections taken from peripheral lymphoid organs of individuals free from infection or inflammation. They observed in regions neighboring the splenic MZ that neutrophils were found in abundance. The aforementioned distribution is observed in both mice and macaques, which implied that these neutrophils around the MZ might be consequential in the maintenance of homeostasis. Moreover, in pathological spleens this distribution changes, such that neutrophils penetrate the germinal centres and follicular mantle. The confinement of neutrophils to the area around the MZ signifies that they are in an optimal location to react to circulating antigens and also leave them in adjacent to MZ B-cells. These B-cells are usually linked with antibody responses that are T-cell independent. In light of this, the authors showed that splenic neutrophils differ from those in circulation in such that in MZ B-cells they are able to moderate activation 
of IgM secretion. Subsequently these cells were termed B-helper neutrophils (NBH), and an in depth analysis of this population unveiled the possible molecular mechanism by which they regulate MZ B-cell activation. Compared to general circulating neutrophils, expression of B-cell stimulating molecules such as APRIL, BAFF, Interleukin-21 and CD40L, are significantly up regulated in NBH. Furthermore, activation of MZ B-cells can occur in medium conditioned with NBH cells, an effect that is annulled when signaling is blocked through these receptors. However, activation of MZ B-cells seems to be influenced by contactdependent mechanisms as well since greater antibody secretion is observed after incubation with NBH cells. Interestingly, unlike general circulating neutrophils, the NBH community impulsively forms neutrophil extracellular trap (NET) like projections containing DNA. Lead better and colleagues [64] proposed that NETs might serve as a potential source of toll-like receptor 9 ligand containing immune complexes, which might facilitate activation of B-cells. Nevertheless, the identification of a NBH cell population (able to acts as proficient helper cells for specifically MZ B-cells) unveils an intriguing new avenue for the correspondence between the adaptive and innate immune branches.

\section{B-CELL-NEUTROPHIL INTERACTION (INFECTION)}
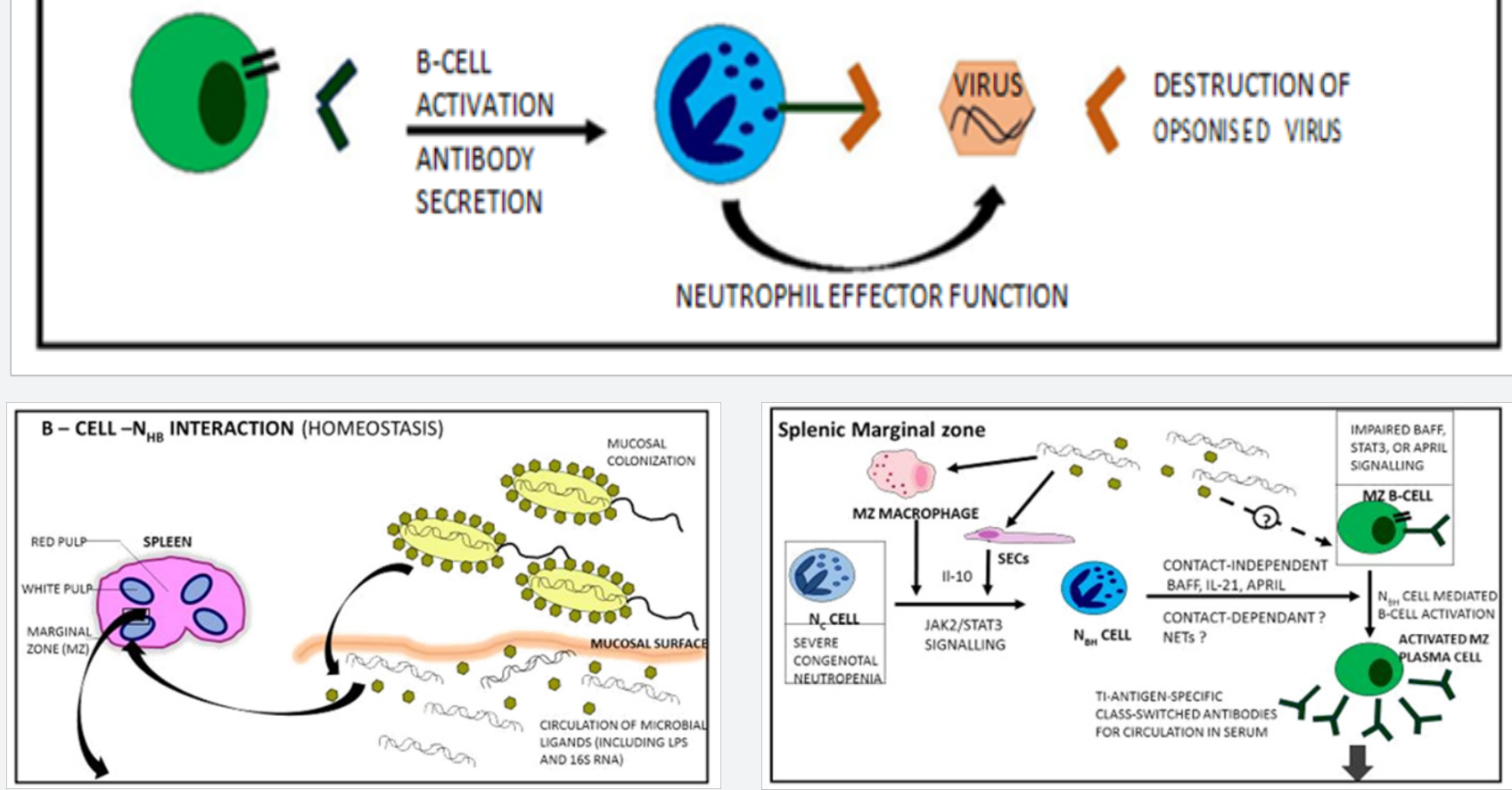

Figure 2: Interaction between B cells and neutrophils. (A) Upon infection, B cells (green) secrete antibodies that coat microbes. Neutrophils (blue) opsonise these coated pathogens. (B) Splenic marginal zone (MZ, pink) B cells (green) are activated to secrete antibodies by the newly identified B-helper neutrophil subset (NBH, dark blue) against T-cell-independent antigens. This activation is mediated by the secretion of IL21, BAFF and APRIL in a contact-independent mechanism. Neutrophil extracellular traps (NETs) and contact-dependant mechanisms might also play a part. Circulatory neutrophils (Nc) give rise to NBH. This occurs through JAK2 and STAT3 signaling in response to IL-10 secretion by sinusoidal endothelial cells (SECs) and/or macrophages. Adapted from [64].

How is the MZ B-cell population affected by this NBHmediated support? The activation of follicular B-cells following T-cell dependent antigen presentation is generally partnered with the development of germinal centre's, this has been adequately described [65]. Germinal centre's have commonly been associated with the process of somatic hyper mutation (SHM) which generates a plethora of Ig genes and results in an assemblage of high-affinity clones as well as the development of immunological memory. Nonetheless, even though it has been shown that during a systemic infection, CD11c(lo) dendritic cells encourage the development of IgM-secreting plasma blasts from MZ B-cells [49], considerably less is known about the influence of helper cell assistance on the initiation of T-cell independent immune responses. This study by Puga et al. [4] demonstrated that the expression of transcription factors such as XBP1 and Blimp1 as well as surface marker CD38 in MZ B-cells, is triggered by NBH cells. The expression of these factors is an indication of the formation of plasma blasts. Moreover, as expression of AID (a 
DNA-editing enzyme necessary for class-switch recombination and somatic hyper mutation) is up regulated in MZ B-cells in the vicinity of NBH cells, class switching was shown to have occurred in secreted antibodies, with generation of IgA and IgG2 being favored. Notably, even though in individuals with severe congenital neutropenia (abnormally low neutrophil count), levels of class-switched antibodies in response to T-cell dependent antigens were normal. Decreased levels of IgG and IgA in response to T-cell independent antigens, like lipopolysaccharides (LPS) were observed. Sequencing indicated that, at least in humans, NBH-activated MZ B-cell secreted antibodies acquire mutations similar to those observed during somatic hypermutation. Interestingly, the assistance from NBHcells seem to trigger antibody expansion from MZ B-cells, this effect is similar to that of CD4+ T-cells on follicular B-cells. The origin of these NBH cells comes into question, given their ability to mediate class-switched antibody secretion from MZ B-cells. When general circulating neutrophils are exposed to interleukin-10, they become inducible NBH-like cells and expression of APRIL and BAFF is up regulated. STAT3 and JAK2 signaling is required for the generation of this inducible population. Sinusoidal epithelial cells, in response to microbial peptides, secrete a variety of neutrophil-attracting chemokines as well as interleukin-10. These sinusoidal epithelial cells are found close to NBH cells in the splenic MZ. In light of this, Puga et al. [4] postulated that microbial ligands, entering the circulation by translocation across microbial surfaces [66], prompt both chemotactic signals to and reprogramming of circulating neutrophils which result in the generation of a NBH population. In agreement with this, splenic NBH cells are established early in fetal life, but only about two days after birth is this population significantly enhanced, this event coincides with the bacterial colonization of mucosal surfaces. Furthermore, mice that are either unable to generate toll-like receptor signaling or are born germ free, have a decreased NBH population. These observations suggest that given a healthy and functional immune system, NBH cells stimulate the formation of class-switched antibodies from MZ B-cells in response to T-cell independent microbial antigens under steady state conditions.

\section{The neutrophil response to tuberculosis infection}

Various studies show definitive evidence that neutrophils are essential to the protective immune response against M.tb infection [17,57,67]. Martineau and colleagues [68] provided evidence that neutropenia led to a considerable decline in both BCG and M. tb levels in whole blood. Numerous studies adequately showed that neutrophils play a major role in the immunopathology during pulmonary tuberculosis infection, with some stating that they are noxious to the host's control of the mycobacterial infection [54-56]. Another study showed that following intratracheal infection, a TB susceptible mouse strain had surprisingly high levels of neutrophils accumulating in the lungs for extended periods [69]. In comparison to less susceptible strains, they demonstrated prolonged existence and lower expression of the CD95 apoptotic receptor associated with greater mobility and phagocytic proficiency of $M$. $t b$. The study concluded that the development of immunopathology during tuberculosis infection was driven by the above mentioned features as well as the fact that/ compared to macrophages, neutrophils battle to control mycobacterial growth [70]. In recent years it became apparent that neutrophils are the main cells infected with actively replicating mycobacteria [71]. This adds to the intricacy of the part they play in M.tb infection, indicating that they act as a concealed stratagem during host infection with $M$. $t b$.

Neutrophils have a broad spectrum of anti-microbial actions. One such action involves the use of human neutrophil peptides (HNPs) which form part of the defensin family of anti-microbial proteins [72]. Mice infected with M. tb H37Rv have shown a significant reduction in the bacillary load in liver spleen and lungs after time and dose dependent treatment with HNP-1 [73]. Martineau et al. [53] also illustrated that HNPs 1-3 kill M. $t b$ in microbiological media. Neutrophils produce lipocalin 2 and cathelicidin LL-37, these peptides both have the ability to restrict mycobacterial growth, with lipocalin 2 acting in an iron dependent manner [74]. This evidence indicates that neutrophils play a considerable part in the host defence during innate immunity. Antimicrobial peptide production facilitates this defense.

During inflammation neutrophils actively produce and secrete a serine proteinase known as ELA2 or leukocyte elastase. It forms part of the chymotrypsin family and has significant microbicidal activity by killing target bacteria and destroying host tissue [75]. ELA2 comprises of three amino acid residues; histidine, aspartate and serine, which are involved in a charge, relay system. This charge relay system allows for the proteinase activity. Within the primary polypeptide these residues are dispersed throughout, it is only once the protein has folded and its three dimensional conformation is complete, that these residues form a triad capable of proteinase activity [76]. Azurophil granules contain ELA2. The function of this elastase is to hydrolyse wide variety of proteins inside the azurophil granules as well as proteins in the extracellular matrix, following its release from the azurophil granules. Neutrophil elastase kills gram-negative bacteria [77], facilitates NET production [78] and degrades bacterial virulence factors [77]. When activated, neutrophil elastase translocates to the nucleus where it partially digests certain histones, promoting chromatin decondensation and leading to NET formation [79]. Myeloperoxidase (MPO), in the presence of ROS such as hydrogen peroxide, had persistent microbicidal action against M.tb H37Rv [79]. When activated, granulocytes such as neutrophils, utilize the myeloperoxidase$\mathrm{H} 2 \mathrm{O} 2-\mathrm{Cl}-$ system to generate reactive aldehydes [80] which at the site of inflammation, covalently alter both membranous and soluble proteins of cells [81]. Also, MPO acts in conjunction with neutrophil elastase to independently drive chromatin decondensation from the elastase's enzymatic activity [78]. 
Lactoferrin, a multifunctional immune protein present in a range of secretory fluids is also contained within the secondary granules of polymorphonuclear leukocytes such as neutrophils [82]. A further study [83] demonstrated the antibacterial capabilities of human lactoferrin early on. This is achieved by lactoferrin sequestering iron from the environment, making this vital element unattainable to potential pathogens [84]. Lactoferrin functions as an adjunct adjuvant in BCG vaccine efficacy and this leads to a boost in protection against future trials with virulent M. tb. An increase in the production of IL-12(p40) as well as an increase in relative ratios of IL-12/IL-10 was observed in mice after a single immunization with lactoferrin [84], this would, in turn, result in the increased recruitment and production of neutrophils.

Neutrophils extracellular traps (NETs) also demonstrate a unique mechanism for the control of infections with intracellular mycobacteria. The formation of NETs is brought about by cellular changes induced by $M$. $t b$, resulting in neutrophil death and subsequent release of $M . t b$ [24]. Even though NETs are able to capture mycobacteria after their release, they are not able to eradicate them. In vivo mycobacterial control could be facilitated by the trapping of $M$. $t b$ by NETs thus restricting distribution of mycobacteria and limiting the infection to the local environment only. A different function for the NET-mediated trapping of $M . t b$ is presumably the sequestering of local chemokines and cytokines, thus initiating granuloma development by promoting recruitment of other phagocytes. Since only a proportion of neutrophils undergo NET formation, the remaining neutrophils are believed to play a role in phagocytosis and other neutrophilic obligations [24]. Even though reactive oxygen species are produced, the combined action with NETs does not eradicate mycobacteria as successful as other microbes [24,85]. It is suggested that NETs bind to the electron dense layer on the outermost structure of mycobacteria. This structure is composed of polysaccharides with their negatively charged groups exposed $[86,87]$.

Neutrophils are capable of undergoing apoptosis $[12,13,58]$. After phagocytosis by neutrophils, mycobacteria are exposed to a variety of antibacterial substances within the intracellular environment of the neutrophil. If these bactericidal substances fail to successfully eradicate the microbe, the neutrophil induces apoptosis in a final effort to eliminate the phagocytosed bacteria. It has also been proposed that in an attempt to be spared from the immune response, mycobacteria induce apoptosis in neutrophils after infection [88-91].

TNF- $\alpha$ is one cytokine responsible for inducing apoptosis in neutrophils [92]. This was illustrated in vitro [93] that neutrophils stimulated with TNF- $\alpha$ and bacterial lipopolysaccharide (LPS) resulted in apoptosis. The study also suggested that apoptosis might be the cause for the low viability observed with neutrophils, and that the obliteration of these neutrophils, unsuccessful in their attempt to kill the mycobacteria, tips the scale towards the infiltration of mononuclear cells rather than neutrophils, during the alteration of the inflammatory immune response against $\mathrm{M}$. tb infection. They also proposed that the rapid cell death of neutrophils at the site of infection might be attributed to M. $t b$ [94].

\section{Concluding Remarks}

Neutrophils are traditionally convicted of being destructive, they arrive on the scene too early, in colossal numbers, and their reaction is unmerciful and usually leads to immunopathology. As more discoveries are made regarding these innate immune cells, however, neutrophils are beginning to emerge as influential mediators between innate and adaptive immune branches and crucial for an effective immune response against pathogens. They function as effectors with a plethora of cytotoxic constituents at their disposal, as well as preparing the micro environment for the more specific adaptive response by secreting the required chemokines and cytokines. They instruct monocytes, dendritic cells and other lymphocytes and serve as a direct connection between innate and humoral immune responses. They greatly influence the decision to initiate, alter or maintain a specific immune response. Being the devoted front line fighters that they are, they fight in life and even in death and come prepared with an arsenal of weaponry against pathogens such as $M$. $t b$, employ kamikaze tactics by apoptosis and form NETs through specialized cell death.

\section{Future Research on the Role of Neutrophils}

Uncovering the full complexity of the mechanism by which neutrophils operate proves to remain a challenge. Unanswered questions that still remain include:

A. The source of the initial signal that initiates formation of the NBH population and

B. The specific regulatory mechanism by which NBHmediated MZ B-cells are activated. Answers to these questions could lead to potential therapeutic advances in the enhancement of basal immunity by the calculated manipulation of neutrophils.

\section{References}

1. Sepkowitz KA (2001) AIDS--the first 20 years. N Engl J Med 344(23): 1764-1772.

2. Donoghue HD, Spigelman M, Greenblatt CL, Lev Maor G, Bar Gal GK, et al. (2004) Tuberculosis: from prehistory to Robert Koch, as revealed by ancient DNA. Lancet Infect Dis 4(9): 584-592.

3. Team EC for DP and C (ECDC)-HCU-E editorial (2013) WHO publishes Global tuberculosis report 2013 Eurosurveillance 18(43): 1.

4. Puga I, Cols M, Barra CM, He B, Cassis L, et al. (2012) B cellhelper neutrophils stimulate the diversification and production of immunoglobulin in the marginal zone of the spleen. Nat Immunol 13(2): 170-180.

5. Borregaard N, Sehested M, Nielsen BS, Sengelov H, Kjeldsen L (1995) Biosynthesis of granule proteins in normal human bone marrow cells. Gelatinase is a marker of terminal neutrophil differentiation. Blood 85(3): 812-817. 
6. Martin C, Burdon PCE, Bridger G, Gutierrez-Ramos JC, Williams TJ, et al. (2003) Chemokines acting via CXCR2 and CXCR4 control the release of neutrophils from the bone marrow and their return following senescence. Immunity 19(4): 583-593.

7. Sibille Y, Reynolds HY (1990) Macrophages and Polymorphonuclear Neutrophils in Lung Defense and Injury. Am Rev Respir Dis 141(2): 471-501.

8. Fujii G, Selsted ME, Eisenberg D (1993) Defensins promote fusion and lysis of negatively charged membranes. Protein Sci Publ Protein Soc 2(8): 1301-1312.

9. Cociancich S, Ghazi A, Hetru C, Hoffmann JA, Letellier L (1993) Insect defensin, an inducible antibacterial peptide, forms voltage-dependent channels in Micrococcus luteus. J Biol Chem 268(26): 19239-19245.

10. Szyk A, Wu Z, Tucker K, Yang D, Lu W, et al. (2006) Crystal structures of human $\alpha$-defensins HNP4, HD5, and HD6. Protein Sci 15(12): 27492760 .

11. Yang D, Chen Q, Chertov O, Oppenheim JJ (2000) Human neutrophil defensins selectively chemoattract naive $\mathrm{T}$ and immature dendritic cells. J Leukoc Biol 68(1): 9-14.

12. Ullah Khan A, Wilson T (1995) Reactive oxygen species as cellular messengers. Chem Biol 2(7):437-445.

13. Lundqvist-Gustafsson H, Bengtsson T (1999) Activation of the granule pool of the NADPH oxidase accelerates apoptosis in human neutrophils. J Leukoc Biol 65(2): 196-204.

14. Hellstrand K, Asea A, Dahlgren C, Hermodsson S (1994) Histaminergic regulation of NK cells. Role of monocyte-derived reactive oxygen metabolites. J Immunol 153(11): 4940-4947.

15. Halliwell B, Gutteridge JM (1990) Role of free radicals and catalytic metal ions in human disease: an overview. Methods Enzymol 186: $1-85$.

16. Pillay J, den Braber I, Vrisekoop N, Kwast LM, de Boer RJ, et al. (2010) In vivo labeling with $2 \mathrm{H} 20$ reveals a human neutrophil lifespan of 5.4 days. Blood 116(4): 625-627.

17. Moulding DA, Quayle JA, Hart CA, Edwards SW (1998) Mcl-1 expression in human neutrophils: regulation by cytokines and correlation with cell survival. Blood 92(7): 2495-2502.

18. Abadie V, Badell E, Douillard P, Ensergueix D, Leenen PJM, et al. (2005) Neutrophils rapidly migrate via lymphatics after Mycobacterium bovis BCG intradermal vaccination and shuttle live bacilli to the draining lymph nodes. Blood 106(5): 1843-1850.

19. Fang FC (2007) Nitric Oxide and Infection. Springer Science \& Business Media pp. 524.

20. Sawant KV, Cho H, Lyons M, Ly LH, McMurray DN (2010) Guinea pig neutrophil-macrophage interactions during infection with Mycobacterium tuberculosis. Microbes Infect 12(11): 828-837.

21. Zhang Y, Broser M, Cohen H, Bodkin M, Law K, et al. (1995) Enhanced interleukin-8 release and gene expression in macrophages after exposure to Mycobacterium tuberculosis and its components. J Clin Invest 95(2): 586-592.

22. Laan M, Cui ZH, Hoshino H, Lötvall J, Sjöstrand M, et al. (1999) Neutrophil recruitment by human IL-17 via C-X-C chemokine release in the airways. J Immunol 162(4): 2347-2352.

23. Hedlund S, Persson A, Vujic A, Che KF, Stendahl O, et al. (2010) Dendritic cell activation by sensing Mycobacterium tuberculosis-induced apoptotic neutrophils via DC-SIGN. Hum Immunol 71(6): 535-540.

24. Silva MT, Silva MN, Appelberg R (1989) Neutrophil-macrophage cooperation in the host defence against mycobacterial infections. Microb Pathog 6(5): 369-380.
25. Ramos-Kichik V, Mondragón-Flores R, Mondragón-Castelán M, Gonzalez-Pozos S, Muñiz-Hernandez S, et al. (2009) Neutrophil extracellular traps are induced by Mycobacterium tuberculosis. Tuberculosis (Edinb) 89(1): 29-37.

26. Buchanan JT, Simpson AJ, Aziz RK, Liu GY, Kristian SA, et al. (2006) DNase expression allows the pathogen group A Streptococcus to escape killing in neutrophil extracellular traps. Curr Biol CB 16(4): 396-400.

27. Beiter K, Wartha F, Albiger B, Normark S, Zychlinsky A, et al. (2006) An endonuclease allows Streptococcus pneumoniae to escape from neutrophil extracellular traps. Curr Biol 16(4): 401-407.

28. Brinkmann V, Reichard U, Goosmann C, Fauler B, Uhlemann Y, et al. (2004) Neutrophil extracellular traps kill bacteria. Science 303(5663): 1532-1535.

29. Fuchs TA, Abed U, Goosmann C, Hurwitz R, Schulze I, et al. (2007) Novel cell death program leads to neutrophil extracellular traps. J Cell Biol 176(2): 231-241.

30. Neufert C, Pai RK, Noss EH, Berger M, Boom WH, et al. (2001) Mycobacterium tuberculosis 19-kDa lipoprotein promotes neutrophil activation. J Immunol 167(3): 1542-1549.

31. Alemán M, Schierloh P, de la Barrera SS, Musella RM, Saab MA, et al. (2004) Mycobacterium tuberculosis Triggers Apoptosis in Peripheral Neutrophils Involving Toll-Like Receptor 2 and p38 Mitogen Protein Kinase in Tuberculosis Patients. Infect Immun 72(9): 5150-5158.

32. Godaly G, Young DB (2005) Mycobacterium bovis bacille Calmette Guerin infection of human neutrophils induces CXCL8 secretion by MyD88-dependent TLR2 and TLR4 activation. Cell Microbiol 7(4): 591-601.

33. Subrahmanyam YV, Yamaga S, Prashar Y, Lee HH, Hoe NP, et al. (2001) RNA expression patterns change dramatically in human neutrophils exposed to bacteria. Blood 97(8): 2457-2468.

34. Kobayashi SD, Voyich JM, Buhl CL, Stahl RM, DeLeo FR (2002) Global changes in gene expression by human polymorphonuclear leukocytes during receptor-mediated phagocytosis: Cell fate is regulated at the level of gene expression. Proc Natl Acad Sci 99(10): 6901-6906.

35. Nathan C (2006) Neutrophils and immunity: challenges and opportunities. Nat Rev Immunol 6(3): 173-182.

36. Scapini P, Lapinet-Vera JA, Gasperini S, Calzetti F, Bazzoni F, et al. (2000) The neutrophil as a cellular source of chemokines. Immunol Rev 177: 195-203.

37. Yang D, de la Rosa G, Tewary P, Oppenheim JJ (2009) Alarmins Link Neutrophils and Dendritic Cells. Trends Immunol 30(11): 531-537.

38. Serhan CN (2007) Resolution Phase of Inflammation: Novel Endogenous Anti-Inflammatory and Proresolving Lipid Mediators and Pathways. Annu Rev Immunol 25(1): 101-137.

39. Van Gisbergen KP, Sanchez-Hernandez M, Geijtenbeek TBH, van Kooyk Y (2005) Neutrophils mediate immune modulation of dendritic cells through glycosylation-dependent interactions between Mac-1 and DCSIGN. J Exp Med 201(8): 1281-1292.

40. Palucka K, Banchereau J (2002) How dendritic cells and microbes interact to elicit or subvert protective immune responses. Curr Opin Immunol 14(4): 420-431.

41. Bennouna S, Bliss SK, Curiel TJ, Denkers EY (2003) Cross-talk in the innate immune system: neutrophils instruct recruitment and activation of dendritic cells during microbial infection. J Immunol 171(11): 6052-6058.

42. Costantini C, Calzetti F, Perbellini O, Micheletti A, Scarponi C, et al. (2011) Human neutrophils interact with both 6-sulfo LacNAc+ DC and 
NK cells to amplify NK-derived IFN $\gamma$ : role of CD18, ICAM-1, and ICAM3. Blood 117(5): 1677-1686.

43. Marucha PT, Zeff RA, Kreutzer DL (1990) Cytokine regulation of IL-1 beta gene expression in the human polymorphonuclear leukocyte. J Immunol 145(9): 2932-2937.

44. Cooper AM, Mayer-Barber KD, Sher A (2011) Role of innate cytokines in mycobacterial infection. Mucosal Immunol 4(3): 252-260.

45. Cicco NA, Lindemann A, Content J, Vandenbussche P, Lübbert $M$, et al. (1990) Inducible production of interleukin- 6 by human polymorphonuclear neutrophils: role of granulocyte-macrophage colony-stimulating factor and tumor necrosis factor-alpha. Blood 75(10): 2049-2052

46. Mackay CR (1996) Chemokine receptors and T cell chemotaxis. J Exp Med 184(3): 799-802.

47. McColl SR, Paquin R, Ménard C, Beaulieu AD (1992) Human neutrophils produce high levels of the interleukin 1 receptor antagonist in response to granulocyte/macrophage colony-stimulating factor and tumor necrosis factor alpha. J Exp Med 176(2): 593-598.

48. Dinarello CA, Cannon JG, Wolff SM, Bernheim HA, Beutler B, et al (1986) Tumor necrosis factor (cachectin) is an endogenous pyrogen and induces production of interleukin 1. J Exp Med 163(6): 1433-1450.

49. Balázs M, Martin F, Zhou T, Kearney J (2002) Blood dendritic cells interact with splenic marginal zone B cells to initiate T-independent immune responses. Immunity 17(3): 341- 352.

50. Dayer JM, Beutler B, Cerami A (1985) Cachectin/tumor necrosis factor stimulates collagenase and prostaglandin E2 production by human synovial cells and dermal fibroblasts. J Exp Med 162(6): 2163-2168.

51. Bachwich PR, Chensue SW, Larrick JW, Kunkel SL (1986) Tumor necrosis factor stimulates interleukin-1 and prostaglandin E2 production in resting macrophages. Biochem Biophys Res Commun 136(1): 94-101

52. Bertolini DR, Nedwin GE, Bringman TS, Smith DD, Mundy GR (1986) Stimulation of bone resorption and inhibition of bone formation in vitro by human tumour necrosis factors. Nature 319(6053): 516-518.

53. Torti FM, Dieckmann B, Beutler B, Cerami A, Ringold GM (1985) A macrophage factor inhibits adipocyte gene expression: an in vitro model of cachexia. Science 229(4716): 867-869.

54. Elias JA, Gustilo K, Baeder W, Freundlich B (1987) Synergistic stimulation of fibroblast prostaglandin production by recombinant interleukin 1 and tumor necrosis factor. J Immunol Baltim Md 1950 138(11): 3812-3816.

55. Kasama T, Strieter RM, Standiford TJ, Burdick MD, Kunkel SL (1993) Expression and regulation of human neutrophil-derived macrophage inflammatory protein 1 alpha. J Exp Med 178(1): 63-72.

56. Lyons MJ, Yoshimura T, McMurray DN (2002) Mycobacterium bovis BCG Vaccination Augments Interleukin-8 mRNA Expression and Protein Production in Guinea Pig Alveolar Macrophages Infected with Mycobacterium tuberculosis. Infect Immun 70(10): 5471-5478.

57. Ahrens B, Gruber C, Rha R-D, Freund T, Quarcoo D, et al. (2009) BCG priming of dendritic cells enhances T regulatory and Th1 function and suppresses allergen-induced Th2 function in vitro and in vivo. Int Arch Allergy Immunol 150(3): 210-220.

58. Shigenaga T, Dannenberg AM, Lowrie DB, Said W, Urist MJ, et al (2001) Immune responses in tuberculosis: antibodies and CD4CD8 lymphocytes with vascular adhesion molecules and cytokines (chemokines) cause a rapid antigen-specific cell infiltration at sites of bacillus Calmette-Guérin reinfection. Immunology 102(4): 466-479.

59. Keane J, Balcewicz-Sablinska MK, Remold HG, Chupp GL, Meek BB, et al. (1997) Infection by Mycobacterium tuberculosis promotes human alveolar macrophage apoptosis. Infect Immun 65(1): 298-304.
60. Theilgaard-Mönch K, Porse BT, Borregaard N (2006) Systems biology of neutrophil differentiation and immune response. Curr Opin Immunol 18(1): 54-60.

61. Chen K, Xu W, Wilson M, He B, Miller NW, et al. (2009) Immunoglobulin D enhances immune surveillance by activating antimicrobial, proinflammatory and B cell-stimulating programs in basophils. Nat Immunol 10(8): 889-898.

62. Chu VT, Fröhlich A, Steinhauser G, Scheel T, Roch T, et al. (2011) Eosinophils are required for the maintenance of plasma cells in the bone marrow. Nat Immunol 12(2): 151-159.

63. Harwood NE, Barral P, Batista FD (2012) Neutrophils the unexpected helpers of B-cell activation. EMBO Rep 13(2): 93-94.

64. Zhang X, Majlessi L, Deriaud E, Leclerc C, Lo-Man R (2009) Coactivation of Syk Kinase and MyD88 Adaptor Protein Pathways by Bacteria Promotes Regulatory Properties of Neutrophils. Immunity 31(5): 761771.

65. Leadbetter EA, Rifkin IR, Hohlbaum AM, Beaudette BC, Shlomchik MJ, et al. (2002) Chromatin-IgG complexes activate B cells by dual engagement of IgM and Toll-like receptors. Nature 416(6881): 603607.

66. MacLennan IC (1994) Germinal centers. Annu Rev Immunol 12: 117139.

67. Clarke TB, Davis KM, Lysenko ES, Zhou AY, Yu Y, et al. (2010) Recognition of peptidoglycan from the microbiota by Nod1 enhances systemic innate immunity. Nat Med 16(2): 228-231.

68. Blomgran R, Ernst JD (2011) Lung neutrophils facilitate activation of naive antigen-specific CD4+ cells during Mycobacterium tuberculosis infection. J Immunol 186(12): 7110-7119.

69. Martineau AR, Newton SM, Wilkinson KA, Kampmann B, Hall BM, et al. (2007) Neutrophil-mediated innate immune resistance to mycobacteria. J Clin Invest 117(7): 1988-1994.

70. Lowe DM, Redford PS, Wilkinson RJ, O'Garra A, Martineau AR (2012) Neutrophils in tuberculosis: friend or foe? Trends Immunol 33(1): 1425.

71. Saunders BM, Britton WJ (2007) Life and death in the granuloma: immunopathology of tuberculosis. Immunol Cell Biol 85(2): 103-111.

72. Duarte TA, Noronha-Dutra AA, Nery JS, Ribeiro SB, Pitanga TN, et al. (2012) Mycobacterium tuberculosis-induced neutrophil ectosomes decrease macrophage activation. Tuberculosis (Edinb) 92(3): 218-225.

73. Eruslanov EB, Lyadova IV, Kondratieva TK, Majorov KB, Scheglov IV, et al. (2005) Neutrophil responses to Mycobacterium tuberculosis infection in genetically susceptible and resistant mice. Infect Immun 73(3): 1744-1753.

74. Eum SY, Kong JH, Hong MS, Lee YJ, Kim JH, et al. (2010) Neutrophils Are the Predominant Infected Phagocytic Cells in the Airways of Patients with Active Pulmonary TB. Chest 137(1): 122-128.

75. Ganz T, Oren A, Lehrer RI (1992) Defensins: microbicidal and cytotoxic peptides of mammalian host defense cells. Med Microbiol Immunol 181(2): 99-105.

76. Sharma S, Verma I, Khuller GK (2001) Therapeutic potential of human neutrophil peptide 1 against experimental tuberculosis. Antimicrob Agents Chemother 45(2): 639- 640 .

77. Sonawane A, Jyot J, During R, Ramphal R (2006) Neutrophil Elastase, an Innate Immunity Effector Molecule, Represses Flagellin Transcription in Pseudomonas aeruginosa. Infect Immun 74(12): 6682-6689.

78. Papayannopoulos V, Metzler KD, Hakkim A, Zychlinsky A (2010) Neutrophil elastase and myeloperoxidase regulate the formation of neutrophil extracellular traps. J Cell Biol 191(3): 677-691. 
79. Borelli V, Banfi E, Perrotta MG, Zabucchi G (1999) Myeloperoxidase Exerts Microbicidal Activity against Mycobacterium tuberculosis. Infect Immun 67(8): 4149-4152.

80. Hazen SL, Hsu FF, d'Avignon A, Heinecke JW (1998) Human neutrophils employ myeloperoxidase to convert alpha-amino acids to a battery of reactive aldehydes: a pathway for aldehyde generation at sites of inflammation. Biochemistry 37(19): 6864-6873.

81. Hazen SL, Gaut JP, Hsu FF, Crowley JR, d'Avignon A, et al. (1997) p-Hydroxyphenylacetaldehyde, the Major Product of l-Tyrosine Oxidation by the Myeloperoxidase-H202-Chloride System of Phagocytes, Covalently Modifies $\varepsilon$-Amino Groups of Protein Lysine Residues. J Biol Chem 272(27): 16990-16998.

82. Sokolov AV, Zakahrova ET, Kostevich VA, Samygina VR, Vasilyev VB (2014) Lactoferrin, myeloperoxidase, and ceruloplasmin: complementary gearwheels cranking physiological and pathologica processes. Biometals 27(5): 815-828.

83. Arnold RR, Cole MF, McGhee JR (1977) A bactericidal effect for human lactoferrin. Science 197(4300): 263-265.

84. Hwang SA, Kruzel ML, Actor JK (2005) Lactoferrin augments BCG vaccine efficacy to generate $\mathrm{T}$ helper response and subsequent protection against challenge with virulent Mycobacterium tuberculosis. Int Immunopharmacol 5(3): 591-599.

85. May ME, Spagnuolo PJ (1987) Evidence for activation of a respiratory burst in the interaction of human neutrophils with Mycobacterium tuberculosis. Infect Immun 55(9): 2304-2307.

86. Paul TR, Beveridge TJ (1992) Reevaluation of envelope profiles and cytoplasmic ultrastructure of mycobacteria processed by conventional embedding and freeze-substitution protocols. J Bacteriol 174(20): 6508-6517.
87. Takade A, Umeda A, Matsuoka M, Yoshida S, Nakamura M, et al. (2003) Comparative studies of the cell structures of Mycobacterium leprae and M. tuberculosis using the electron microscopy freeze-substitution technique. Microbiol Immunol 47(4): 265-270.

88. Suttmann H, Lehan N, Böhle A, Brandau S (2003) Stimulation of Neutrophil Granulocytes with Mycobacterium bovis Bacillus CalmetteGuérin Induces Changes in Phenotype and Gene Expression and Inhibits Spontaneous Apoptosis. Infect Immun 71(8): 4647-4656.

89. Rojas M, Barrera LF, Puzo G, Garcia LF (1997) Differential induction of apoptosis by virulent Mycobacterium tuberculosis in resistant and susceptible murine macrophages: role of nitric oxide and mycobacterial products. J Immunol 159(3): 1352-1361.

90. Molloy A, Laochumroonvorapon P, Kaplan G (1994) Apoptosis, but not necrosis, of infected monocytes is coupled with killing of intracellular bacillus Calmette-Guerin. J Exp Med 180(4): 1499-1509.

91. Hachicha M, Naccache PH, McColl SR (1995) Inflammatory microcrystals differentially regulate the secretion of macrophage inflammatory protein 1 and interleukin 8 by human neutrophils: a possible mechanism of neutrophil recruitment to sites of inflammation in synovitis. J Exp Med 182(6): 2019-2025.

92. Osamu Hachiya YT (1995) Inhibition by bacterial lipopolysaccharide of spontaneous and TNF-alpha-induced human neutrophil apoptosis in vitro. Microbiol Immunol 39(9): 715-723.

93. Kasahara K, Sato I, Ogura K, Takeuchi H, Kobayashi K, et al. (1998) Expression of Chemokines and Induction of Rapid Cell Death in Human Blood Neutrophils by Mycobacterium tuberculosis. J Infect Dis 178(1): 127-137.

94. Tiku K, Tiku ML, Skosey JL (1986) Interleukin 1 production by human polymorphonuclear neutrophils. J Immunol 136(10): 3677-3685.

\section{Your next submission with Juniper Publishers} will reach you the below assets

- Quality Editorial service

- Swift Peer Review

- Reprints availability

- E-prints Service

- Manuscript Podcast for convenient understanding

- Global attainment for your research

- Manuscript accessibility in different formats ( Pdf, E-pub, Full Text, Audio)

- Unceasing customer service

Track the below URL for one-step submission https://juniperpublishers.com/online-submission.php 